\title{
Focusing a NIR adaptive optics imager; experience with GSAOI
}

Matthew Doolan, Gabe Bloxham, Peter Conroy, Damien Jones, Peter McGregor, et al.

Matthew Doolan, Gabe Bloxham, Peter Conroy, Damien Jones, Peter McGregor, Dejan Stevanovic, Jan Van Harmelen, Liam E. Waldron, Mark Waterson, Ross Zhelem, "Focusing a NIR adaptive optics imager; experience with GSAOI," Proc. SPIE 6273, Optomechanical Technologies for Astronomy, 62732D (6 July 2006); doi: $10.1117 / 12.670891$

Event: SPIE Astronomical Telescopes + Instrumentation, 2006, Orlando, Florida, United States 


\title{
Focusing a NIR Adaptive Optics Imager, Experience with GSAOI
}

\author{
Matthew Doolan ${ }^{\mathrm{a}}$, Gabe Bloxham ${ }^{\mathrm{a}}$, Peter Conroy ${ }^{\mathrm{a}}$, Damien Jones ${ }^{\mathrm{d}}$, Peter McGregor ${ }^{\mathrm{a}}$, Dejan \\ Stevanovic $^{\mathrm{b}}$, Jan Van Harmelen ${ }^{\mathrm{a}}$, Liam E. Waldron ${ }^{\mathrm{c}}$, Mark Waterson ${ }^{\mathrm{a}}$, Ross Zhelem ${ }^{\mathrm{a}}$ \\ ${ }^{a}$ Research School of Astrophysics and Astronomy, Australian National University, Mount Stromlo \\ Observatory, Cotter Rd Weston, ACT, 2611, Australia. \\ ${ }^{b}$ Electro-Optic Systems Pty Ltd, 111 Canberra Ave, Griffith, ACT, 2603, Australia. \\ ${ }^{c}$ Locata Corporation, 401 Clunies Ross St, Acton, ACT, 2601, Australia. \\ ${ }^{\mathrm{d}}$ Prime Optics, 17 Crescent Rd, Eumundi, QLD, 4562, Australia.
}

\begin{abstract}
The Gemini South Adaptive Optics Imager (GSAOI) to be used with the Multi-Conjugate Adaptive Optics (MCAO) system at Gemini South is currently in the final stages of assembly and testing. GSAOI uses a suite of 26 different filters, made from both BK7 and Fused Silica substrates. These filters, located in a non-collimated beam, work as active optical elements.

The optical design was undertaken to ensure that both the filter substrates both focused longitudinally at the same point. During the testing of the instrument it was found that longitudinal focus was filter dependant. The methods used to investigate this are outlined in the paper. These investigations identified several possible causes for the focal shift including substrate material properties in cryogenic conditions and small amounts of residual filter power.
\end{abstract}

Keywords: near-infrared, instrumentation, Gemini, focus techniques

\section{INTRODUCTION}

The Gemini South Adaptive Optics Imager ${ }^{1}$ (GSAOI) is a near infrared imager designed to operate with the Multi Conjugate Adaptive Optics (MCAO) system at Gemini South. GSAOI uses 4 Hawaii 2-RG ${ }^{4}$ detectors in a mosaic for the focal plane. A suite of 22 filters is provided at delivery, including broadband $\mathrm{Z}, \mathrm{H}, \mathrm{J}$ and $\mathrm{K}$ filters and numerous narrow band filters, with the ability to add an additional 4 filters at a late stage. Guiding is provided with the use of an On Detector Guide Window (ODGW) system, where a small part of the detector can be read out at high rates while not impacting the science integration frame.

GSAOI is required to deliver diffraction limited science images from the MCAO system with extremely high levels of resolution. This requirement means that, when focused, the instrument should not significantly impact on the wave front error of the image received from MCAO. This is quantified in the requirement that no more than 65nm RMS wave front error will be introduced by GSAOI over the wavelength range of $0.9-2.4 \mu \mathrm{m}$.

GSAOI is in the final stages of testing and will be delivered to Gemini in the middle of this year. This paper describes the tools used in determining best focus for the instrument to the stringent requirements, the difficulties experienced and the results achieved.

\section{OPTICAL DESIGN}

GSAOI is designed to work over the wavelength range determined by the sensitivity of the detector which is $900 \mathrm{~nm}-$ $2.5 \mu \mathrm{m}$. This means that the choice of materials available for the design is limited. In relation to the focusing of the instrument there are several different aspects of the design which are important. The filters are located in a noncollimated beam, thereby making them active optical elements. The filters are also made of two different materials, with the short wavelength filters being made from BK7, while the rest are made from infra-red grade fused silica (Infrasil).

The BK7 substrate filters are chosen such that the co-efficient of the thermal expansion of both the coating and the substrate are similar thus ensuring the filter survives thermal cycling. The same substrate material could not be chosen for the $\mathrm{K}$ band filters as BK7 reduces the transmission over this wavelength range. Therefore, the $\mathrm{K}$ band filters were

Optomechanical Technologies for Astronomy, edited by Eli Atad-Ettedgui, Joseph Antebi, Dietrich Lemke, Proc. of SPIE Vol. 6273, 62732D, (2006) · 0277-786X/06/\$15 - doi: 10.1117/12.670891 
designed to use a Infrasil substrate. To compensate for the two different filter materials with different refractive indexes the substrates were designed at different thicknesses, to ensure that the effective path length remains identical. This requires a knowledge of the refractive index of the two materials at cryogenic temperatures. Models of the refractive index as a function of temperature were developed for the two different substrate materials.

The model for Infrasil was based on previous experience from the NIFS ${ }^{2}$ and $\mathrm{NIRI}^{3}$ instruments at temperatures of $60 \mathrm{~K}$ $-80 \mathrm{~K}$. However, no BK7 data was available during the optical design process. The BK7 cryogenic refractive index was carefully estimated and extrapolated by using cryogenic data for SF6 and higher temperature data for other similar glasses in the SCHOTT Glass Catalogue. Unfortunately, the model could not be fine-tuned because no melt data was available for each filter glass. Using these two different models for the refractive index of the materials at GSAOI operating temperatures, the filter substrates were designed to be $6 \mathrm{~mm}$ and $5.5 \mathrm{~mm}$ thick for Infrasil and BK7 respectively.

\section{FOCUS MEASUREMENT TECHNIQUES}

\subsection{Sloped Focus Mask}

Several different techniques were used to determine the point of best focus for GSAOI. All techniques utilized a mask located in the field position of the GSAOI optical path. This mask (Figure 1) incorporated a series of different features including pinholes, separated lines and a Ronchi screen. The mask is tilted with respect to the optical axis which results in one end of the mask being inside focus while the other end is outside focus. The nominal focal position is located at the centre of the mask. Markers on the mask indicate the longitudinal distance from nominal focus measured at the focal plane.

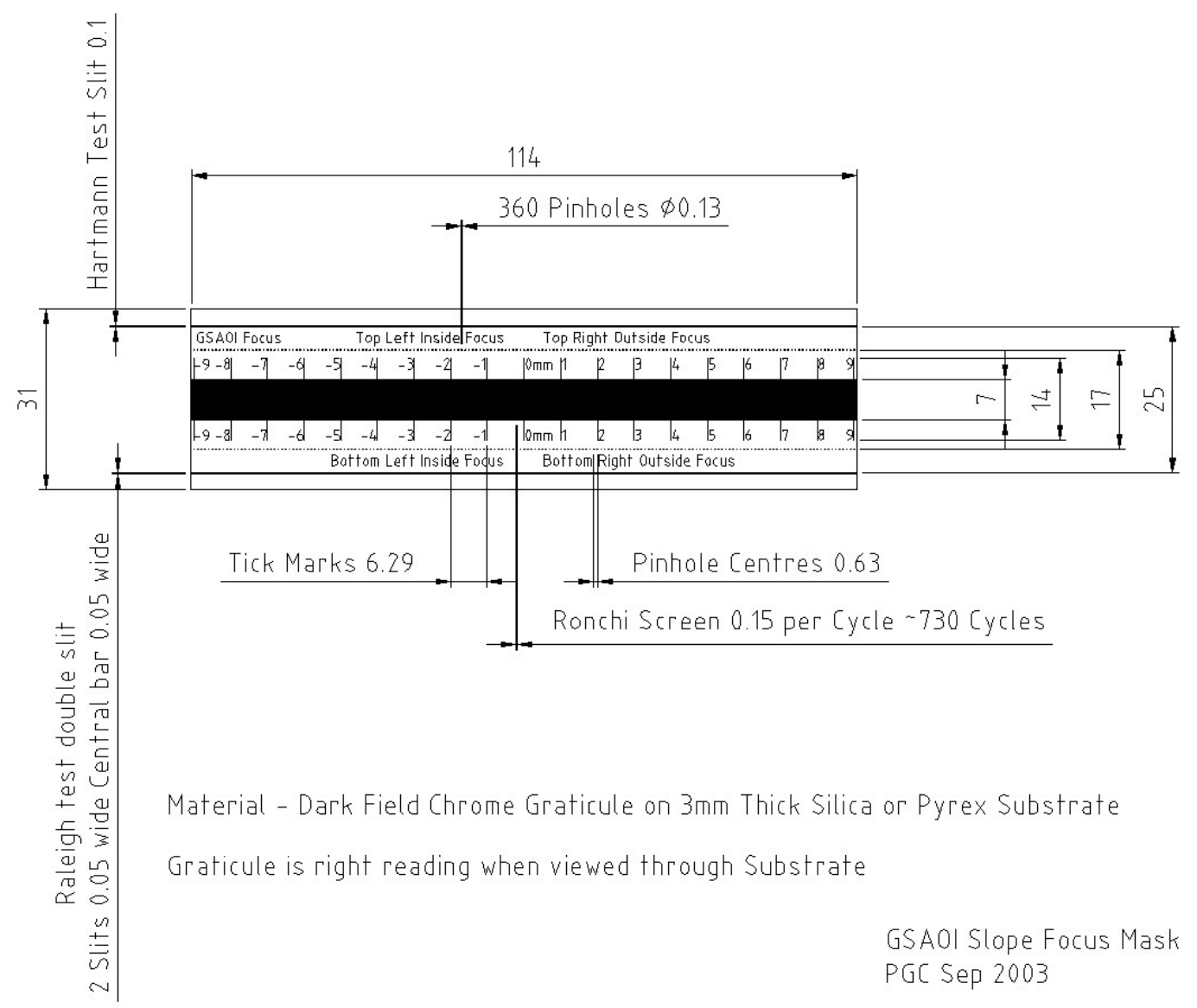

Figure 1: GSAOI sloped focus mask 
The markings on the focal mask are designed to be used in a number of different test configuraions each producing a measurement of the defocus of the detectors. The relationship between focus at the field position and focus at the detector (Figure 2) is clearly a linear relationship.

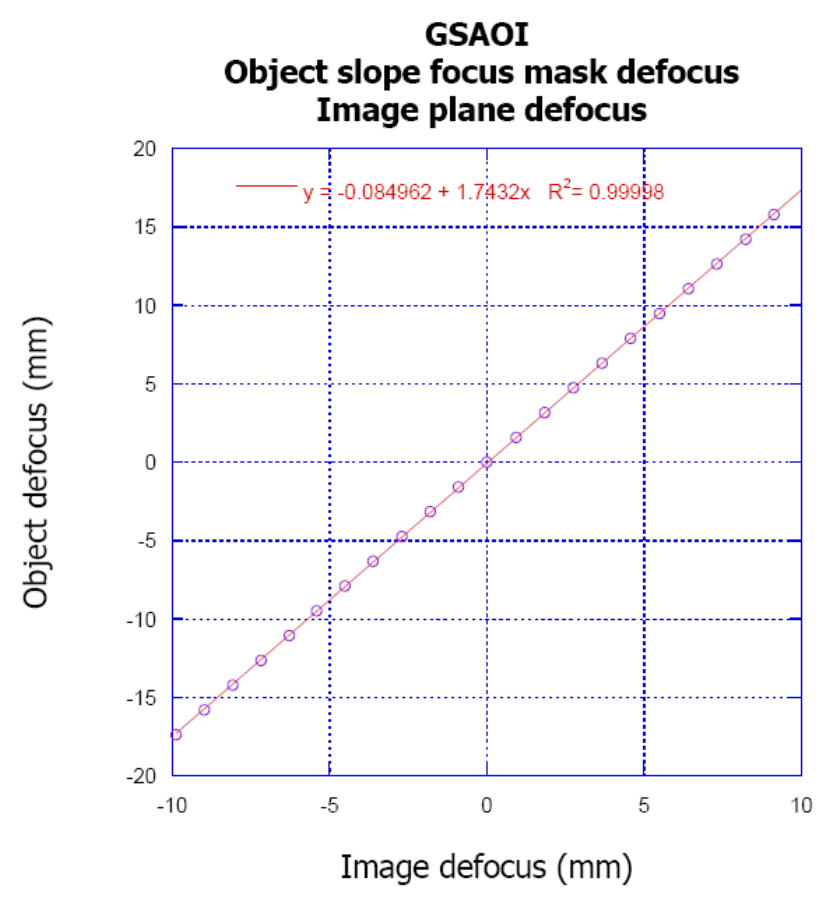

Figure 2: Ratio between field focus and detector focus positions

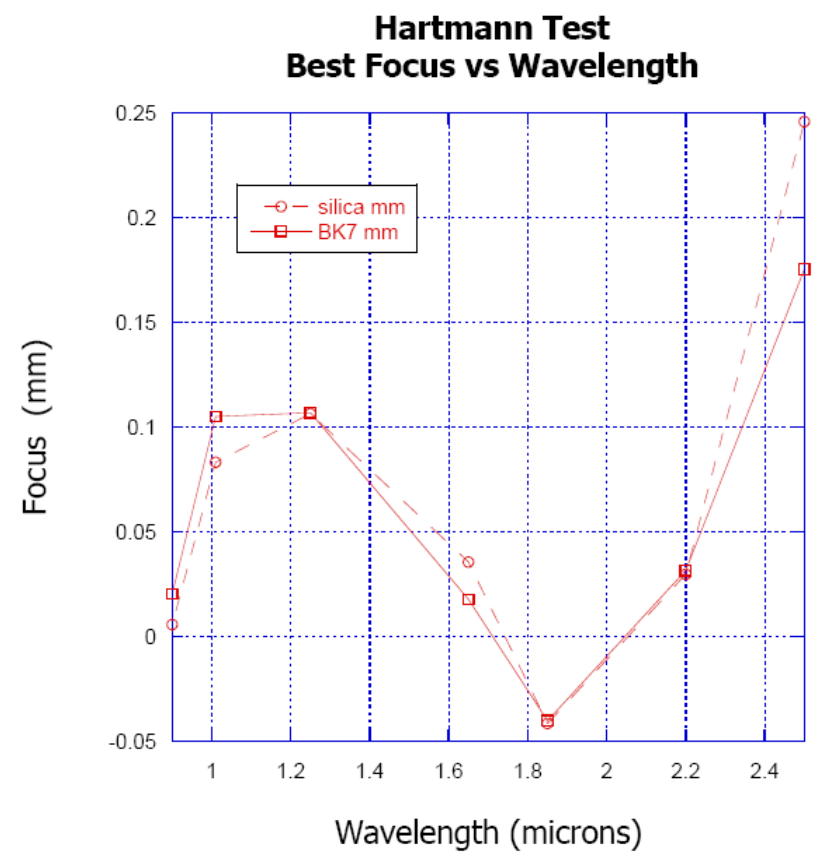

Figure 3: Zemax predicted position of best focus as a function of wavelength without window 
This mask is located inside the cryostat at the apparent point of focus produced by MCAO. This results in an approximation as the window is an active optical element in the GSAOI design. When omitted longitudinal color is introduced. To ensure that this effect is not significant, a Zemax analysis of the system without the window was performed (Figure 3). The depth of field for GSAOI is $0.5 \mathrm{~mm}$ and the identified effect of longitudinal color is about $0.2 \mathrm{~mm}$, which is less than the depth of field, but not sufficiently small to be ignored.

The following sections present the different methods used to measure focus and some of the results achieved.

\subsection{Rayleigh lines}

The lines on the sloped focus mask are separated sufficiently that they distinguishable as two separate lines when at focus. By plotting the intensities of the light across the two lines it is possible to identify the point where the two are distinguishable as separate lines. The separation of the lines was calculated such that in the $\mathrm{H}$ band the lines would only be separable when in focus. This assumes that desirable focus of the instrument is where the point spread function does not noticeably grow. The GSAOI focusing criterion is that it must not significantly impact the wave front produced by MCAO. The images of the separated lines showed a large range over which the lines were clearly distinguishable (Figure 4). A point transition from two separate lines to a single line was also difficult to define. This meant that the method was ineffective in identifying the point of best focus.

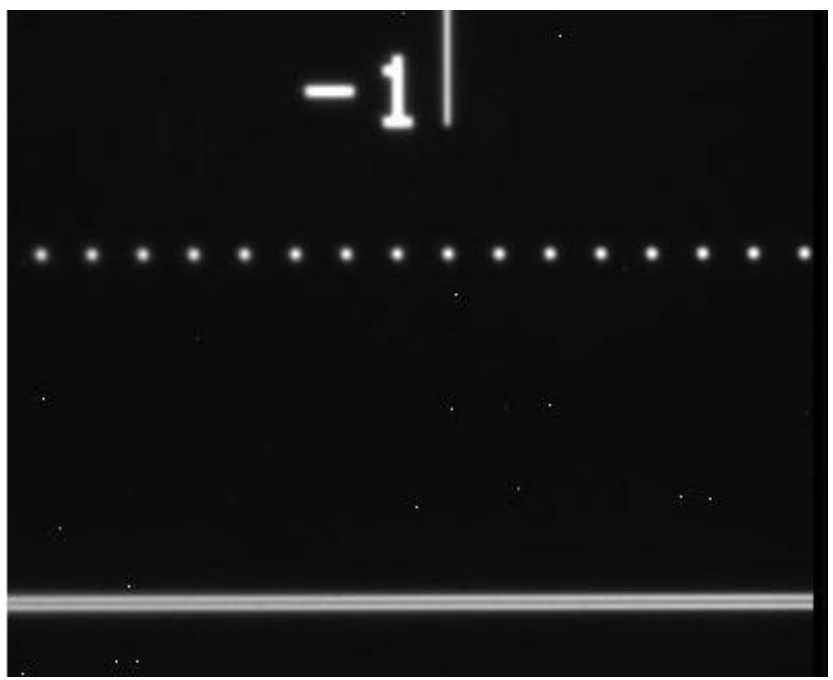

Figure 4: Separated lines are clearly distinguishable over a large focus range

\subsection{Ronchi test}

The Ronchi test utilizes the pattern of alternating light and dark lines on the focal mask. The distance between these lines was calculated to ensure that when the instrument is in focus the light and dark lines are clearly distinguishable. As the lines pass out of focus the distinction between the light and the dark lines is reduced and therefore the intensity difference reduces.

Images were captured for all the filters using this technique and the intensity through these images was plotted (Figure 5) against position. Due to GSAOI having a mosaic of detectors the peak in intensity should be located between the detectors when they are in focus. To identify a point of best focus from this test the first null point on either side of the focus was determined. Best focus was then approximated as being half way between these two points. As can be seen from the graphs shown below, identifying the minima on these graphs was very subjective, as there is no clear point where the amplitude of the beating changes from decreasing to increasing. 


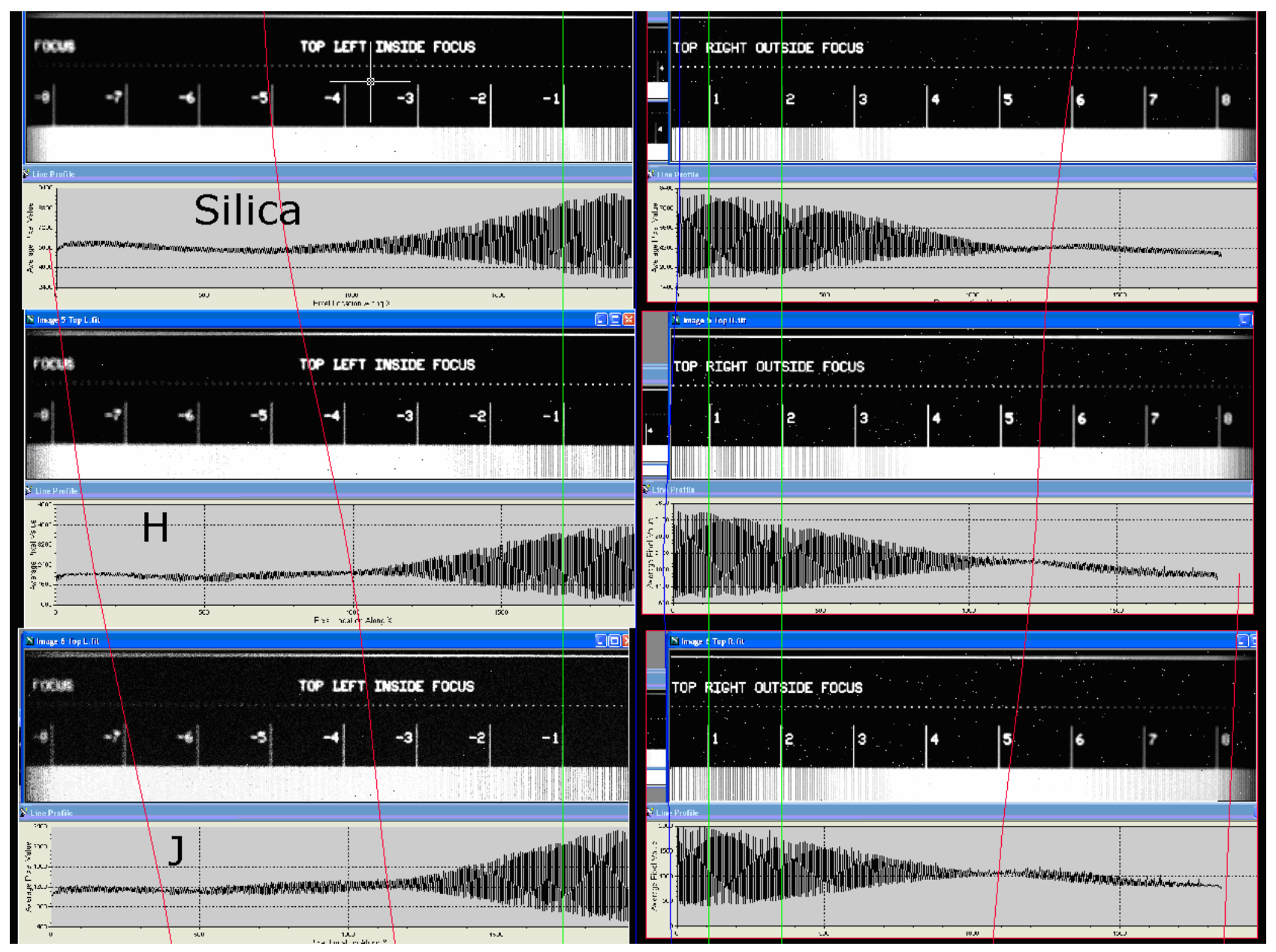

Figure 5: Ronchi test focus results

The measurements made using the Ronchi test were consistent with those achieved using other techniques. However, the subjective nature of this test made it difficult to determine a definitive point of focus.

\subsection{Hartmann masks}

The Hartmann mask test was originally intended to be used with two different markings on the focal mask, the line and the pinholes (Figure 1). Following data capture, only the pinholes were used in the analysis due to the ease of data reduction. The technique relied on projecting a series of pinholes at different focal positions through the system. A Hartmann mask, located in the second filter wheel (Figure 6), was then inserted into the beam near the pupil. This ensured that only one side of the pupil was illuminated when creating the image on the detector. A second mask was then moved into the science beam, allowing the other side of the pupil to be illuminated. This results in two images of points at different focal positions with light passing through the two sides of the pupil. 


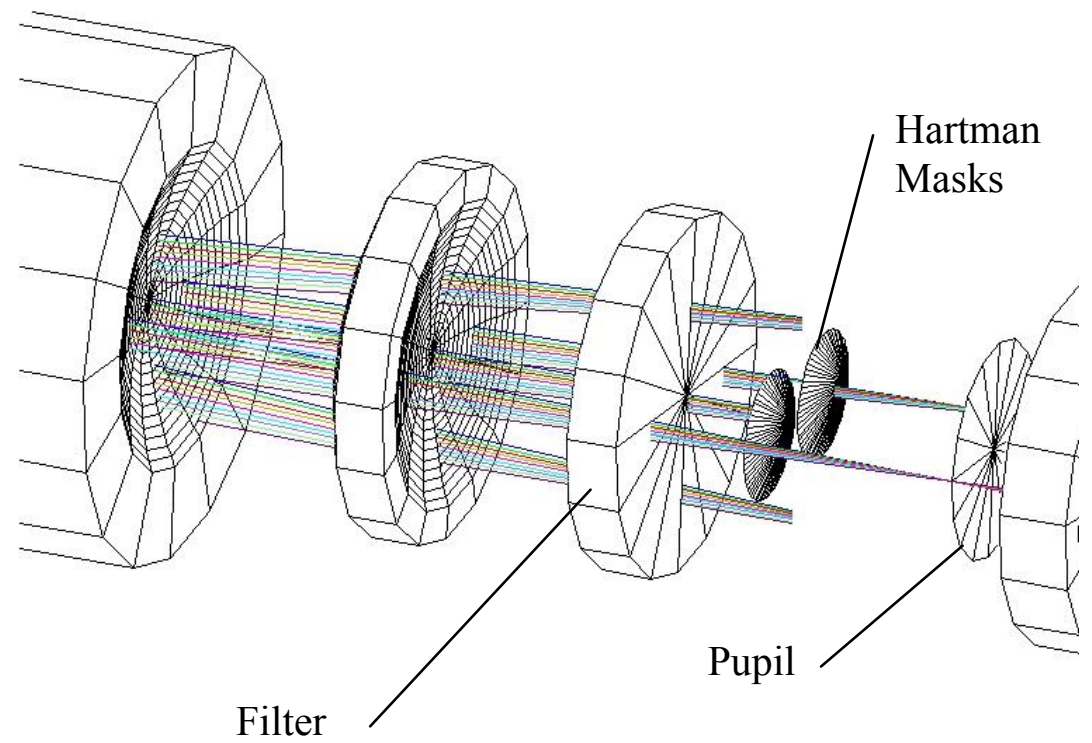

Figure 6: Hartmann mask position in GSAOI

The analysis then involved fitting a line through the pinhole centroids in each image. Each image produced a straight line with a different slope (Figure 7), the intersection of these two lines is at the point of best focus. This analysis was carried out for each of the four detectors in GSAOI. Each detector produced a plane of best focus which was averaged with the remaining three measurements providing a single plane of best focus.
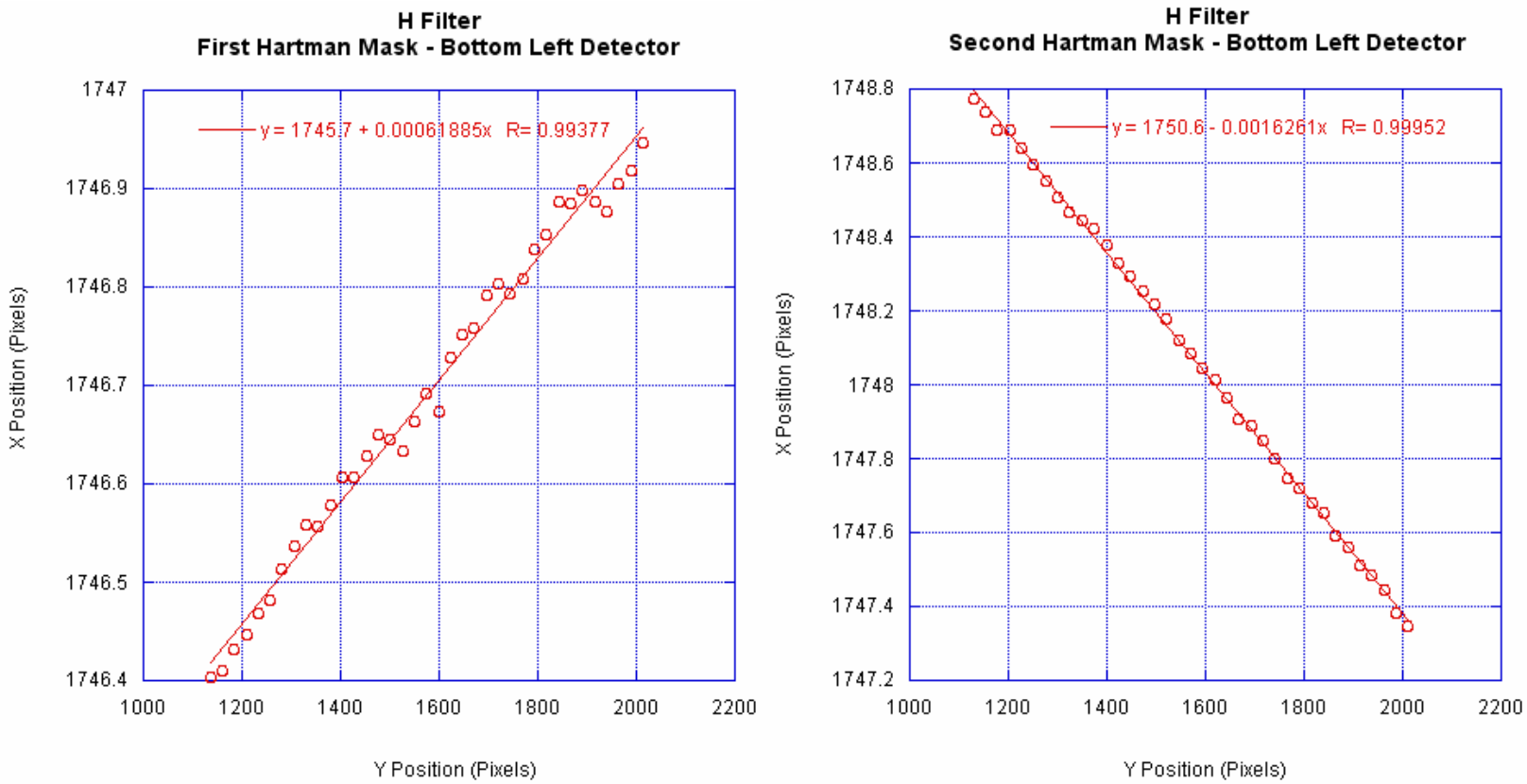

Figure 7: Results from the Hartmann Tests for H-band filter 
The results from the Hartmann Masks are indicated in Table 1. As can be seen, there is a range of different focal positions. This movement in the measured focal position was outside the depth of field of $0.5 \mathrm{~mm}$. Further investigation of these differences in focal position is discussed in Section 4.

Table 1: Filters deviation from nominal focus

\begin{tabular}{|l|c|l|}
\hline Filter & Glass & Focus Position (mm) \\
\hline Z & BK7 & 0.66 \\
\hline J & BK7 & 0.43 \\
\hline H & BK7 & 0.25 \\
\hline K Prime & Infrasil & -0.04 \\
\hline K Short & Infrasil & 0.20 \\
\hline K & Infrasil & 0.00 \\
\hline J Continuum & BK7 & 0.99 \\
\hline H Continuum & BK7 & 0.55 \\
\hline CH4s & BK7 & 0.53 \\
\hline K Short Continuum & Infrasil & 0.48 \\
\hline K Long Continuum & Infrasil & 0.50 \\
\hline
\end{tabular}

The Hartmann test had two approximations to the ideal situation. The first, which has been mentioned previously, is that the window is not included in the measurement. Secondly, the Hartmann masks are not located exactly at a pupil. To ensure that only half of the pupil is illuminated, as required in a Hartmann Test, we have taken an image of the pupil with the each Hartmann Mask in the beam (Figure 8). As can be seen from these images, the Hartmann Masks do indeed illuminate equal and opposite sides of the pupil.
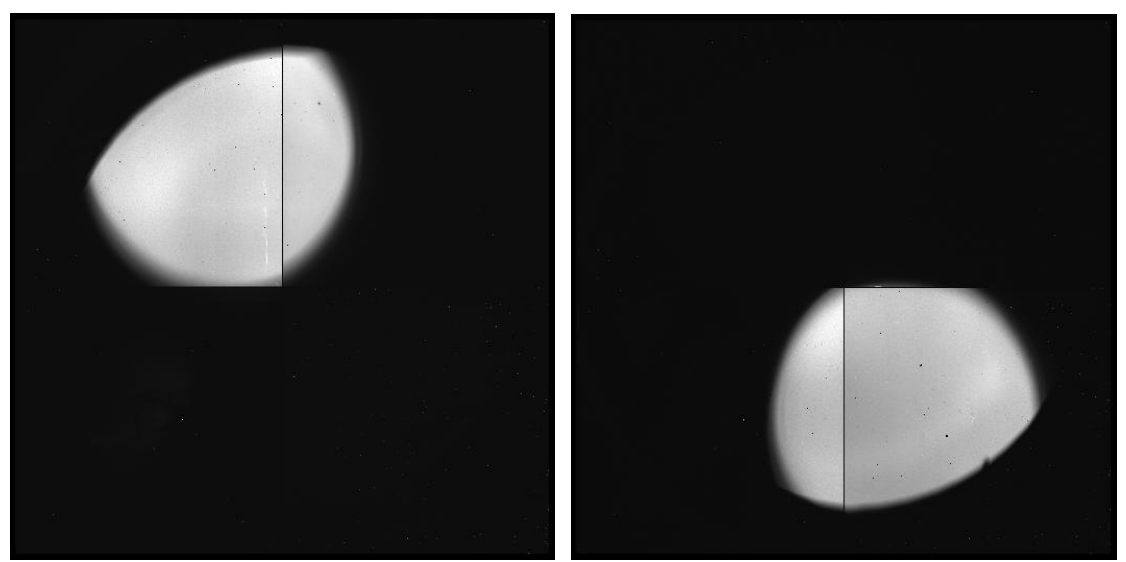

Figure 8: Two images of the pupil with the two Hartmann masks in the beam

\subsection{FWHM}

The final method of determining best focus was the Full Width Half Maximum (FWHM) method. Measurements were taken of the spot size produced by the pinholes in the sloped focus mask. These values were then plotted against position on the detector and distance from nominal focus was inferred. A function was then fitted through these points with the minimum identifying the point of best focus (Figure 9). 


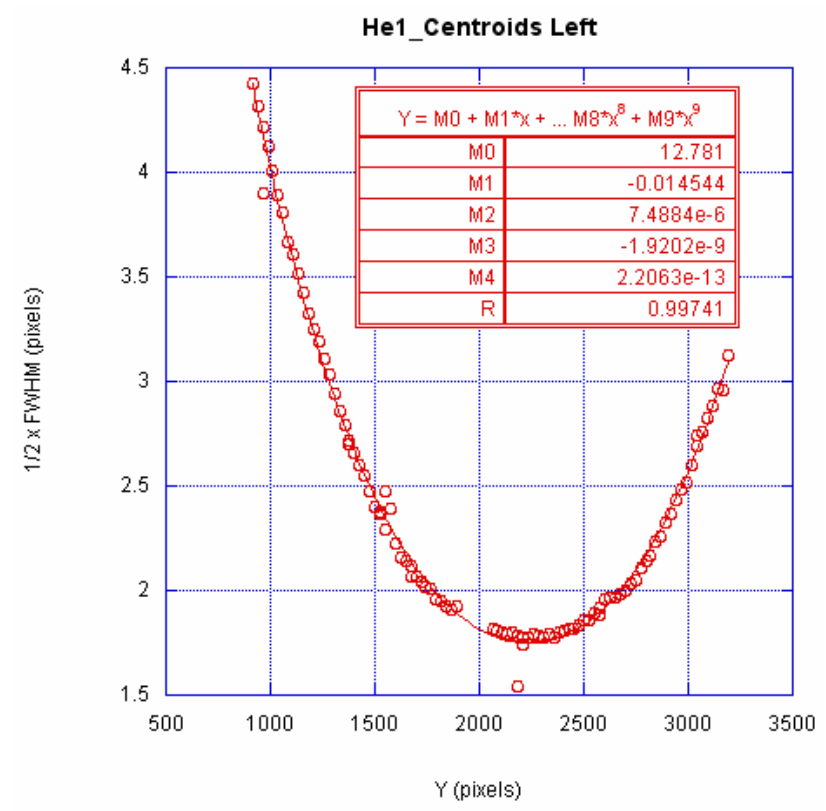

Figure 9: Full Width Half Maximum (FWHM) measurements of pinholes projected from different focal position using a He1 line filter. The point of best focus is shown to be at pixel 2232.

This technique was used for the filters that are located in the same filter wheel as the Hartmann Masks. The results of this analysis provided results similar to those for the Hartmann mask test. Results of the focus measurement are presented in Section 3.4. This method could have been used for all filters, but as the data reduction tools for the Hartmann mask testing had been developed previously this was used for most of the focus measurements.

\section{FILTER SUBSTRATE INVESTIGATION}

As discussed in the previous sections of this paper the measured focal positions for the different filters in GSAOI were outside the allowable variation. To understand the cause of this variation further analysis of the filters and their substrates was undertaken.

The front and rear faces of the filters were measured to identify any power that may be present. This was firstly performed warm with an autocollimator with an adjustable eyepiece. This investigation showed that the filters had small levels of curvature with a small resultant power. However, this was insufficient to result in the measured longitudinal shift in focus. The autocollimator surface curvature measurements where confirmed with the use of an interferometer (Figure 10), which showed the collimator measurements to be accurate to better than $0.3 \mu \mathrm{m}$ of sag.
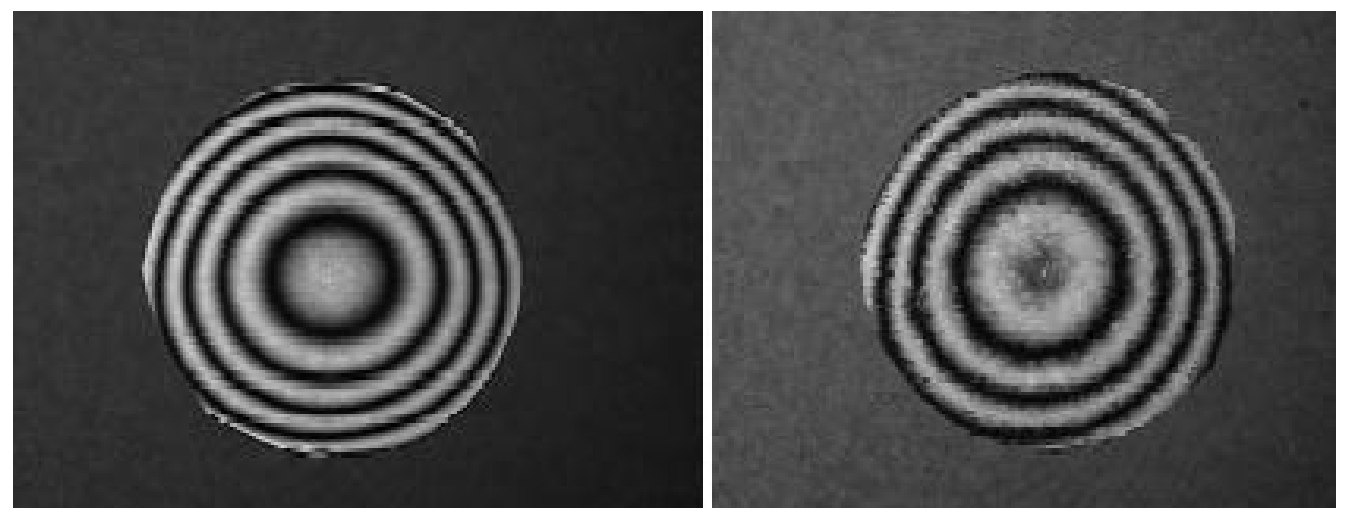

Figure 10: Front and rear face of the $\mathrm{K}$ band filter 
A cold study of the filters then needed to be undertaken to ensure that additional power was not introduced by the thermal stresses. This investigation initially involved the use of Finite Element Analysis methods to predict the stresses experienced in the filter, especially at the interface layer between the coating and the substrate. This showed the shear stresses to be substantial (Figure 11). The simulation did not show any change in thickness between the edges and centre of the filter, indicating no overall power due to this stressing of the material

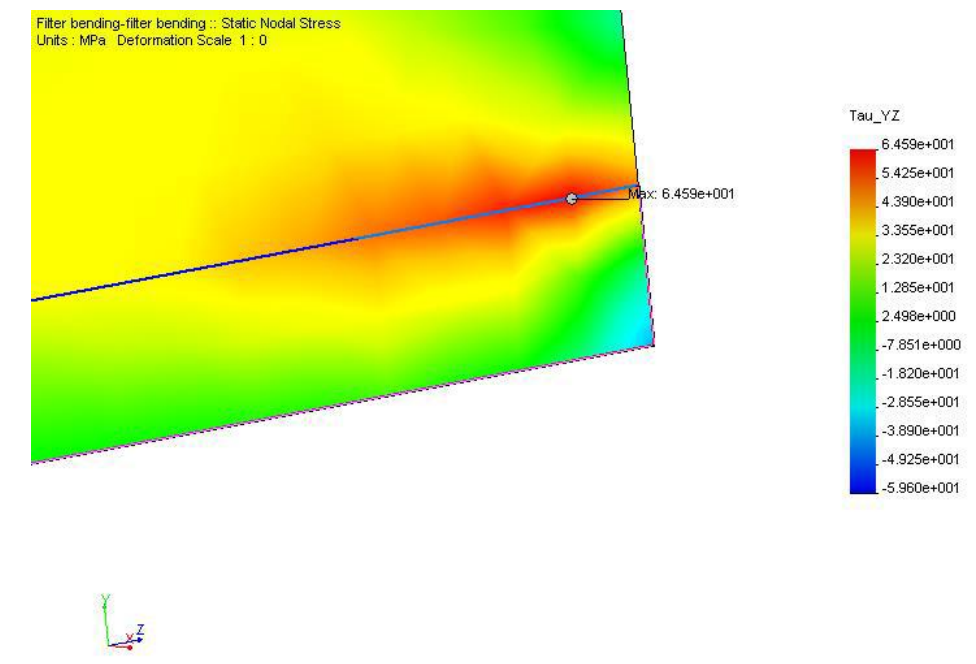

Figure 11: Finite Element Analysis of coating substrate interface stresses

To confirm this analysis, measurements were again taken with the autocollimator, this time with the filters cooled to about $90 \mathrm{~K}$ in a continuous flow liquid nitrogen cryostat. These measurements showed bending induced by the cooling of the filters. However, the filter bent in a meniscus shape with no additional power measured due to the cooling process. This confirmed the Finite Element Analysis results.

Lastly a test of the filter substrates was performed by placing two uncoated pieces of Infrasil and two uncoated pieces of BK7 in the first filter wheel. A Hartmann test was performed on each uncoated substrate. It was noted that the BK7 does not transmit as well above $2.4 \mu \mathrm{m}$. This test involved using white light without any filters, the range of wavelengths measured was determined by the detector sensitivity and the transmission of the optical elements. Due to BK7's lower level of transmission in longer wavelengths it acted as partial filter. This should not have a significant effect on the position of each centroid as the difference in total flux landing on the detector should be small.

The results (Table 2) of this test showed that the BK7 and the Infrasil did indeed focus at different points. Moreover the two different melts of BK7 produced significantly different focus positions, while the Infrasil focus position had very little relation to the batch from which it came. The variation between the two BK7 substrates was greater than the difference in measurement between the both Infrasil substrates and the second of the BK7 substrates.

Table 2: Substrate focus measurements

\begin{tabular}{|l|l|}
\hline Substrate & Focus Position (mm) \\
\hline Infrasil 1 & 0.48 \\
\hline Infrasil 2 & 0.43 \\
\hline BK7 1 & 0.85 \\
\hline BK7 2 & 0.58 \\
\hline
\end{tabular}

\section{CONCLUSIONS}

Each of the four different focus determination methods used in the development of GSAOI gave a measurement of the best point of focus. Two of the mehods were identified as being more effective, the Hartmann mask test and the FWHM test. The Hartmann mask technique was used because of existing data reduction tools. This technique provided a good 
level of accuracy when averaged over a number of different measurements reducing its susceptibility noise. The FWHM test provided results very similar to those achieved with the Hartmann mask.

The design of GSAOI made the filters critical elements in the optical system. Therefore, tight tolerances were required to ensure that the system performed as desired. The substrate material showed significant variation in focus point between different melts of the BK7 material and the different materials. This variation coupled with slight amounts of residual power was identified as the most likely cause for the longitudinal focus shifts for GSAOI.

\section{REFERENCES}

1. P. McGregor, J. Hart, D. Stevanovic, G. Bloxham, D. Jones, J. van Harmelen, J. Griesbach, M. Dawson, P. Young, and M. Jarnyk, "Gemini South Adaptive Optics Imager (GSAOI)", Proc. S.P.I.E., 5492, pp. 1033-1044, 2004.

2. P. McGregor, J. Hart, P. Conroy, L. Pfitzner, G. Bloxham, D. Jones, M. Downing, M. Dawson, P. Young, M. Jarnyk, and J. van Harmelen, "Gemini near-infrared integral field spectrograph (NIFS)", Proc. S.P.I.E., 4841, pp. 1581-1591, 2002.

3. Klaus W. Hodapp, Joseph B. Jensen, Everett M. Irwin, Hubert Yamada, Randolph Chung, Kent Fletcher, Louis Robertson, Joseph L. Hora, Douglas A. Simons, Wendy Mays, Robert Nolan, Matthieu Bec, Michael Merrill, and Albert M. Fowler, “The Gemini Near-Infrared Imager", PASP, 115, 1388.

4. Mark Waterson, Peter McGregor, Jan van Harmelen, Murray Dawson, and Matthew Doolan, "Characterization and Performance of HAWAII-2RG Focal Plane Arrays for NIFS and GSAOI", accepted for publication, Proc S.P.I.E., 2006. 